\title{
Decline in EBV-Specific IFN T cell responses in Kenyan infants from a malaria holoendemic region of Kenya
}

\author{
Amolo S Asito ${ }^{1,2^{*}}$, Erwan Piriou' ${ }^{1}$ Odada P Sumba², Ann M Moormann ${ }^{3}$, Rosemary Rochford ${ }^{1}$ \\ From 13th International Conference on Malignancies in AIDS and Other Acquired Immunodeficiencies \\ (ICMAOI) \\ Bethesda, MD, USA. 7-8 November 2011
}

\section{Background}

Endemic Burkitt's lymphoma, the most prevalent childhood cancer in Equatorial Africa, is a rapidly growing B-cell malignancy that is ultimately fatal if untreated. Two co-factors are linked to the etiology of this pediatric cancer: Epstein-Barr virus (EBV) infection, and sustained and intense exposure to Plasmodium falciparum malaria (holoendemic malaria). In this study, we wanted to test the hypothesis that $P$. falciparum infections during early infancy results in elevated EBV viral load which results in diminished EBV-specific T-cell immune responses over time.

\section{Methods}

Infants were enrolled from two rural sites in Kenya: Kisumu District where malaria transmission is holoendemic and risk for eBL is high and Nandi District where malaria transmission is limited and the risk for eBL is low. Finger prick blood samples were taken through 2 years of age to measure EBV viral load, EBV antibodies, and malaria parasitemia. Venous blood samples were collected at 12, 18 and 24 months of age and PBMC were isolated and stimulated with peptides for both EBV lytic and latent antigens. After 2.5 days of stimulation, IFN $\gamma$ ELISPOTS enumerated EBV-specific T cell responses, and the number of SFU $/ 10^{6}$ PBMC was determined by scanning with ImmunoSpot Reader and Software.

\section{Results}

When we compared EBV lytic and latent IFN $\gamma \mathrm{T}$ cell responses at 12, 18 and 24 months of age, we saw that

\footnotetext{
* Correspondence: jakogwanjo@gmail.com

${ }^{1}$ SUNY Upstate Medical University, Syracuse, NY, USA

Full list of author information is available at the end of the article
}

although children in Kisumu were able to mount an IFNy response against EBV lytic peptides, the magnitude of that response declined significantly by 24 months of age. In contrast, the magnitude of the response did not decline in the Nandi cohort. We also observed higher overall viral loads in infants from Kisumu suggesting that the apparent loss of EBV-specific IFN $\gamma$ response to lytic antigens in the Kisumu children may be associated with these higher viral loads.

\section{Conclusions}

We found that by 2 years of age, there was a significant difference in the capacity of children living in a malaria holoendemic region compared to malaria sporadic region to maintain a $\mathrm{T}$ cell response to EBV lytic antigens. This suggests that $P$. falciparum malaria contributes to loss of EBV-specific immunity by inducing the collapse of an antiviral IFN- $\gamma$ mediated $\mathrm{CD} 8+\mathrm{T}$ cell response.

\section{Acknowledgement}

Funding was provided by R01 CA120667 and D43 CA153701. ASA and EP contributed equally to this work.

\section{Author details}

'SUNY Upstate Medical University, Syracuse, NY, USA. ${ }^{2}$ Center for Global Health Research, Kenya Medical Research Institute, Kisumu, Kenya. ${ }^{3}$ University of Massachusetts Medical School, Worcester, MA, USA.

Published: 19 April 2012

doi:10.1186/1750-9378-7-S1-022

Cite this article as: Asito et al:: Decline in EBV-Specific IFN T cell responses in Kenyan infants from a malaria holoendemic region of Kenya. Infectious Agents and Cancer 2012 7(Suppl 1):O22. 